\title{
Diseño de un sistema de inyección para impresión 3D con sustancia cementosa
}

\author{
DOI: $10.46932 / s f j d v 2 n 1-059$
}

Received in: November 1st, 2020

Accepted in: December 30th, 2020

\author{
Camila Granados Giraldo \\ Ingeniería mecatrónica \\ Universidad Autónoma de Bucaramanga \\ Bucaramanga, Colombia \\ E-mail:mgranados420@unab.edu.co \\ Nelson Higuera Uribe \\ Ingeniería mecatrónica \\ Universidad Autónoma de Bucaramanga \\ Bucaramanga, Colombia \\ E-mail: nhiguera@unab.edu.co \\ Melisa Pineda Fajardo \\ Ingeniería mecatrónica \\ Universidad Autónoma de Bucaramanga \\ Bucaramanga, Colombia \\ E-mail: lpineda485@unab.edu.co
}

\section{RESUMEN}

En el presente artículo se propone el diseño de un prototipo de sistema de inyección de una sustancia cementosa para una impresora 3D implementada en la construcción de viviendas. El prototipo cuenta con una bomba de tornillo o un motor lineal, que es responsable de impulsar el fluido, el cual saldrá de manera uniforme gracias a una válvula reguladora de caudal.

Palabras Clave: Impresión 3D, Sustancia cementosa, Bomba de tornillo, Motor lineal, Válvula reguladora de caudal.

\section{INTRODUCCIÓN}

La industria de la construcción se encuentra entre las más peligrosas. Según Behrokh Khoshnevis, cada año mueren alrededor de 10 mil trabajadores por accidentes y alrededor de 400 se lastiman en el transcurso de su trabajo.

En Colombia las tasas de accidentalidad y mortalidad en esta industria son elevadas; en el 2012 se registraron fatalidades derivadas de la actividad laboral, de las cuales 125 corresponden al sector de la construcción.

Si se hace referencia a otros aspectos, la mano de obra requiere mucho más tiempo para terminar la construcción, considerando que pueden ocurrir inconvenientes e imprevistos durante el proceso. El uso de una impresora 3D favorece a la empresa en el ámbito económico porque agiliza la construcción, minimiza los costos de mano de obra y se reducen considerablemente los errores en la obra. 


\section{OBJETIVO GENERAL}

Diseñar un prototipo de sistema de inyección de una sustancia cementosa para una impresora 3D.

\section{OBJETIVOS ESPECÍFICOS}

- Seleccionar la sustancia con las características más óptimas para el correcto funcionamiento del sistema de impresión.

- Diseñar el sistema de impresión 3D de la sustancia seleccionada.

- Modelar matemáticamente el sistema de posicionamiento de la boquilla teniendo en cuenta su comportamiento dinámico.

\section{METODOLOGÍA}

Para el desarrollo del proyecto se establece una metodología basada en la metodología FourSight de la Universidad de Buffalo.

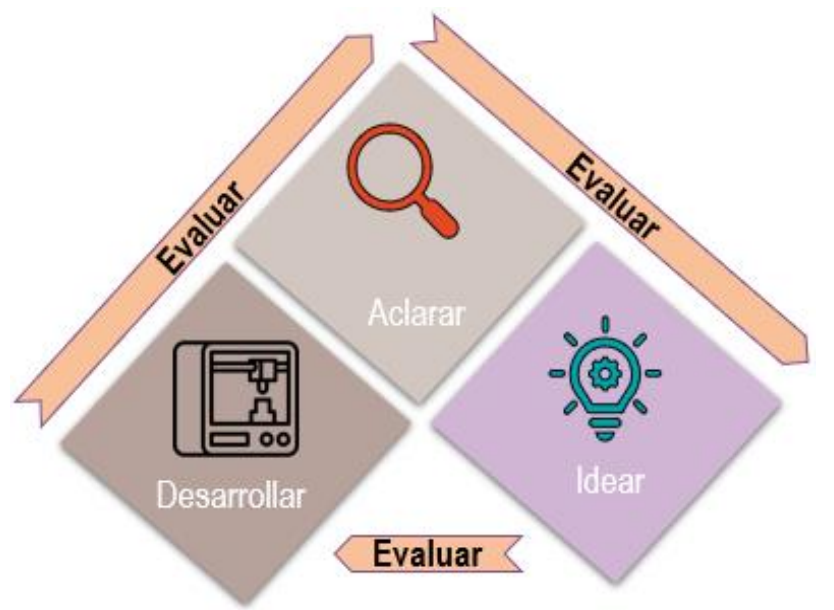

- Aclarar

- Definir las características fundamentales de la impresión 3D para la construcción de viviendas.

- Realizar una búsqueda y recolección de información en torno a la impresión 3D en el área de construcción.

\section{- Idear}

- Idear posibles soluciones que satisfagan los requerimientos del sistema de impresión identificados previamente.

\section{- Desarrollar}

- Diseñar por medio de una herramienta CAD el prototipo del sistema de impresión 3D. 


\section{REFERENTES TEÓRICOS}

\subsection{CONCEPTO:}

\section{- Impresión 3D}

También llamada manufactura aditiva. La impresión 3D es un proceso el cual inicia en un diseño elaborado en un dispositivo de cómputo en donde se especifican la trayectoria del sistema y las capas de material que debe tener el diseño.

La impresión 3D de viviendas tiene algunas ventajas como son la disminución de costos en mano de obra, la disminución de tiempos de fabricación, un menor desperdicio de material y la minimización de fallos.

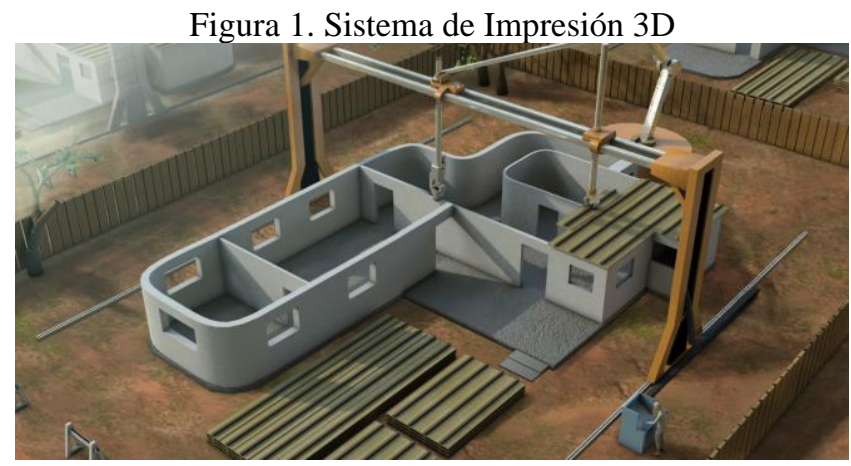

\subsection{SISTEMA FÍSICO:}

\section{- Cilindro Neumático}

Los actuadores neumáticos convierten la energía del aire comprimido en trabajo mecánico generando un movimiento lineal mediante servomotores de diafragma o cilindros, o bien un movimiento giratorio con motores neumáticos. Los cilindros neumáticos de movimiento lineal son utilizados comúnmente en aplicaciones donde la fuerza de empuje del pistón y su desplazamiento son elevados. Entre los mismos se encuentran los cilindros de simple y doble efecto, el cilindro tándem, el de multi posición, el cilindro neumático guiado, el cilindro sin vástago y el cilindro neumático de impacto.

\section{- Válvula reguladora de caudal}

Es una válvula de control que regula el paso de un fluido a través de un circuito hidráulico, permitiendo obtener un caudal de salida constante. Los reguladores se utilizan para gases y líquidos y pueden ser un dispositivo integral con un ajuste de presión de salida, un limitador y un sensor en un solo cuerpo, o consisten en un sensor de presión, controlador y válvula de flujo separados.

\section{- Bomba de tornillo}

Esta bomba utiliza un tornillo helicoidal excéntrico que se mueve dentro de una camisa y hace fluir 
el líquido entre el tornillo y la camisa. Está específicamente indicada para bombear fluidos viscosos, con altos contenidos de sólidos, que no necesitan removerse o que formen espumas si se agitan.

\section{- Servomotor}

Es un dispositivo electromecánico que consiste en un motor eléctrico, un juego de engranes y una tarjeta de control. Un servo tiene la capacidad de ser controlado en posición. Es capaz de ubicarse en cualquier posición dentro de un rango de operación generalmente de $180^{\circ}$ pero puede ser fácilmente modificado para tener un giro libre de $360^{\circ}$. Los servomotores funcionan por medio de modulación de ancho de pulso (PWM), todos los servos disponen de tres cables, dos para alimentación Vcc y Gnd (4.8 a 6 [V]) y un tercero para aplicar el tren de pulsos de control, que hace que el circuito de control diferencial interno ponga el servo en la posición indicada.

\subsection{SUSTANCIA DEL SISTEMA DE IMPRESIÓN:}

\section{- Pegacor}

Es un adhesivo cementico especializado para la instalación de pisos, revestimientos cerámicos y paredes en zonas interiores y exteriores. Cuando el pegacor está mezclado con el agua tiene una consistencia cremosa y tarda de 5 a 10 minutos en secarse. Si la mezcla se encuentra en un recipiente tarda 3 horas en secarse. Entre sus características principales se encuentran:

Tabla 1. Características Pegacor.

\begin{tabular}{|l|l|l|}
\hline \multicolumn{2}{|l|}{ Densidad } & $1.7 \pm 0.05 \mathrm{~g} / \mathrm{cm}^{3}$ \\
\hline Tiempo de ajuste & $20 \mathrm{~min}$ \\
\hline Deslizamiento & $<2 \mathrm{~mm}$ \\
\hline $\begin{array}{l}\text { Consistencia en estado } \\
\text { fresco }\end{array}$ & Cremosa \\
\hline $\begin{array}{l}\text { Relación de agua en la } \\
\text { mezcla }\end{array}$ & Producto & Agua \\
\cline { 2 - 2 } & $\Rightarrow$ Producto amigable con el ambiente. \\
& $\Rightarrow$ Presenta baja retracción y el fraguado es \\
& $\begin{array}{l}\text { más lento lo que permite reparar gran } \\
\text { cantidad de mezcla y hacer correcciones }\end{array}$ \\
fácilmente. & $\Rightarrow$ Genera bajo polvo durante la preparación. \\
& $\Rightarrow$ Producto fabricado bajo procedimientos \\
& ISO 9001 y 14001. \\
\hline
\end{tabular}

\section{RESULTADOS ESPERADOS}

- Diseño del sistema de impresión 3D elaborado mediante una herramienta CAD.

- Modelo matemático del sistema de posicionamiento de la boquilla para la impresión 3D. 


\section{SOLUCIÓN PLANTEADA}

Se plantea como solución el siguiente diseño del sistema de impresión 3D. El cual consiste en un brazo robótico de 6 grados de libertad, los cuales se indican en la figura 2 .

Por medio de un brazo robótico, se puede controlar la posición de la boquilla, y de esta manera generar el movimiento requerido para realizar el diseño que se necesita.

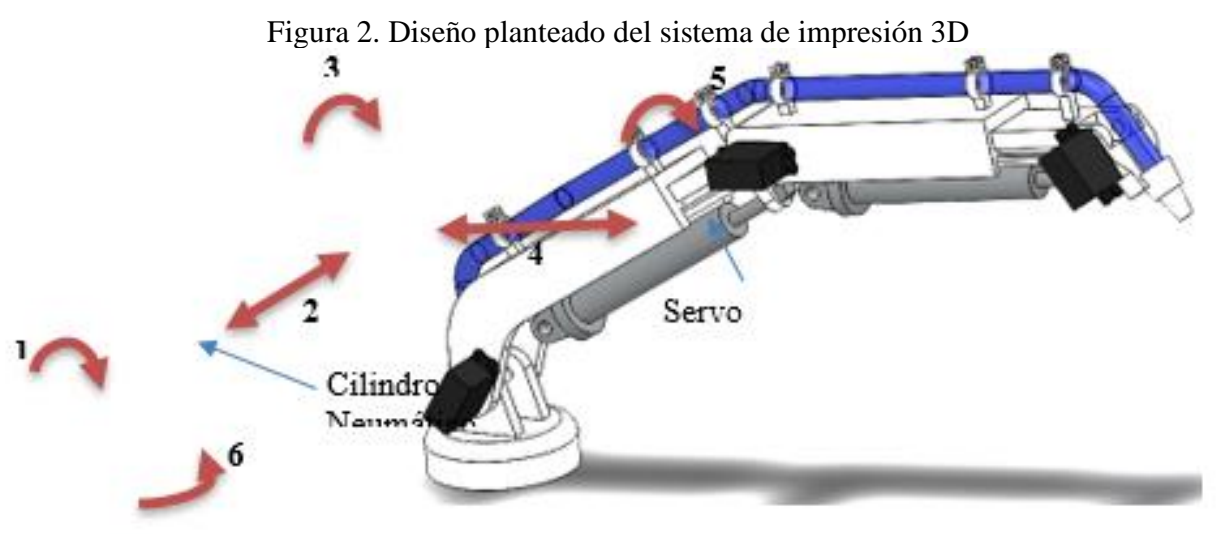

\section{MODELADO MATEMATICO}

Figura 3. Diagrama del brazo robótico

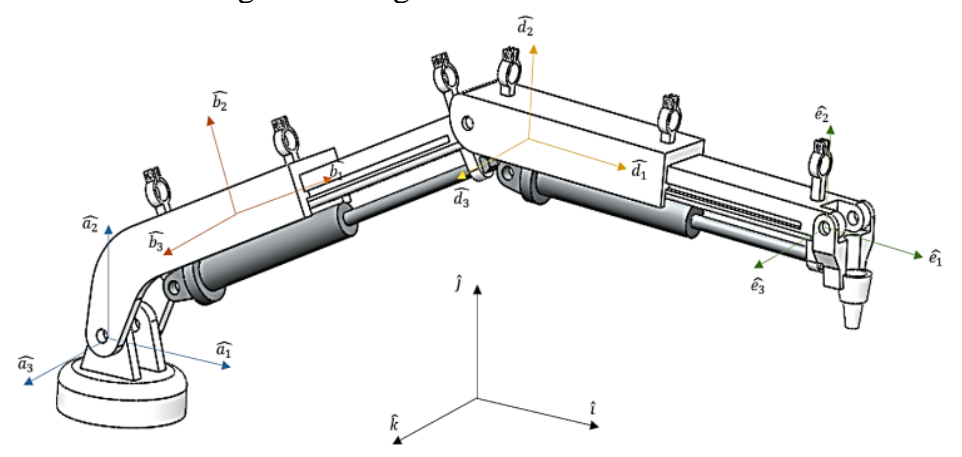

A continuación se presentan las matrices de rotación que describen el movimiento de cada uno de los eslabones que conforman el sistema propuesto.

Ecuación 1. Matriz de rotación del eslabón A con respecto al sistema de coordenadas natural.

$$
\begin{gathered}
i j k-a_{1} a_{2} a_{3} \\
C
\end{gathered}=\left[\begin{array}{ccc}
c \theta & 0 & -s \theta \\
0 & 1 & 0 \\
s \theta & 0 & c \theta
\end{array}\right]
$$


Ecuación 2. Matriz de rotación del eslabón B con respecto al eslabón A.

$$
\begin{gathered}
\widehat{a_{1}}=c \theta \hat{\imath}-s \theta \hat{k} \\
\widehat{a_{2}}=\hat{\jmath} \\
\widehat{a_{3}}=s \theta \hat{\imath}+c \theta \hat{k} \\
a_{1} a_{2} a_{3} \rightarrow b_{1} b_{2} b_{3}=\left[\begin{array}{ccc}
c \phi & s \phi & 0 \\
-s \phi & c \phi & 0 \\
0 & 0 & 1
\end{array}\right]
\end{gathered}
$$

Ecuación 3. Matriz de rotación del eslabón D con respecto al eslabón B.

$$
\begin{gathered}
\widehat{b_{1}}=c \phi \widehat{a_{1}}+s \phi \widehat{a_{2}} \\
\widehat{b_{2}}=-s \phi \widehat{a_{1}}+c \theta \widehat{a_{2}} \\
\widehat{b_{3}}=\widehat{a_{3}} \\
b_{1} b_{2} b_{3} \rightarrow d_{1} d_{2} d_{3}=\left[\begin{array}{ccc}
c \beta & s \beta & 0 \\
-s \beta & c \beta & 0 \\
0 & 0 & 1
\end{array}\right]
\end{gathered}
$$

Ecuación 4. Matriz de rotación del eslabón E con respecto al eslabón D.

$$
\begin{gathered}
\widehat{d_{1}}=c \beta \widehat{b_{1}}+s \beta \widehat{b_{2}} \\
\widehat{d_{2}}=-s \beta \widehat{b_{1}}+c \beta \widehat{b_{2}} \\
\widehat{d_{3}}=\widehat{b_{3}} \\
d_{1} d_{2} d_{3}-e_{1} e_{2} e_{3}=\left[\begin{array}{ccc}
c \gamma & s \gamma & 0 \\
-s \gamma & c \gamma & 0 \\
0 & 0 & 1
\end{array}\right]
\end{gathered}
$$

Figura 4. Sistema de coordenadas A con respecto al sistema de coordenadas natural

$$
\begin{aligned}
& \widehat{e_{1}}=c \gamma \widehat{d_{1}}+s \gamma \widehat{d_{2}} \\
& \widehat{e_{2}}=-s \gamma \widehat{d_{1}}+c \gamma \widehat{d_{2}} \\
& \widehat{e_{3}}=\widehat{d_{3}}
\end{aligned}
$$

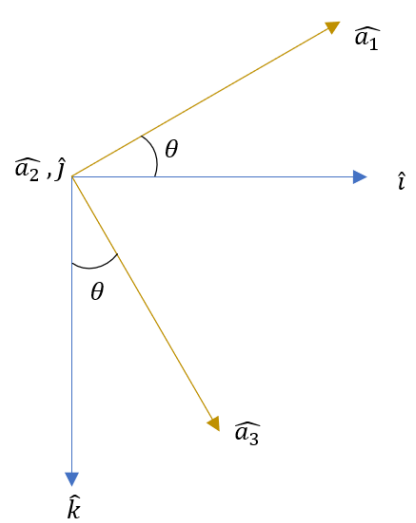


Figura 5. Sistema de coordenadas $B$ con respecto al sistema de coordenadas $A$

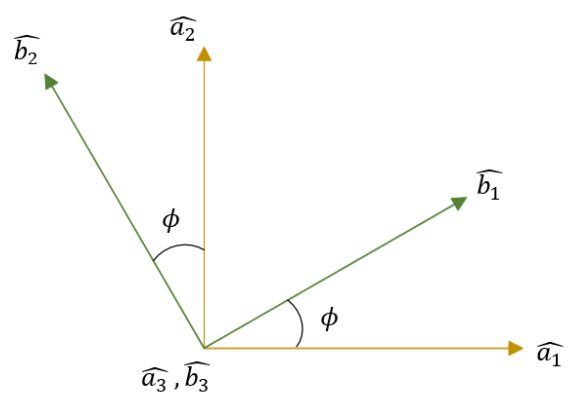

Figura 6. Sistema de coordenadas D con respecto al sistema de coordenadas B

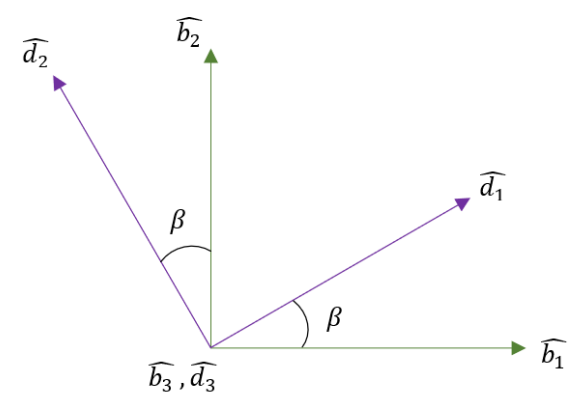

Figura 7. Sistema de coordenadas E con respecto al sistema de coordenadas D

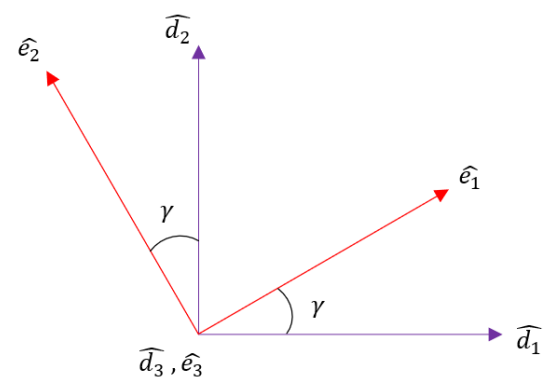

\section{IDENTIFICACIÓN DEL PROYECTO}

\begin{tabular}{|lr|l|}
\hline $\begin{array}{l}\text { Nombre del } \\
\text { Semillero }\end{array}$ & Modelado y simulación \\
\hline Tutor del Proyecto & Sebastian Roa Prada \\
\hline $\begin{array}{l}\text { Grupo de } \\
\text { Investigación }\end{array}$ & GICYM \\
\hline $\begin{array}{l}\text { Línea de } \\
\text { Investigación }\end{array}$ & Modelado y Simulación \\
\hline $\begin{array}{l}\text { Fecha de } \\
\text { Presentación }\end{array}$ & $06 / 10 / 2017$ \\
\hline
\end{tabular}

Este material es presentado al VI Encuentro Institucional de Semilleros de Investigación UNAB, una actividad carácter formativo. La Universidad Autónoma de Bucaramanga se reserva los derechos de divulgación con fines académicos, respetando en todo caso los derechos morales de los autores y bajo discrecionalidad del grupo de investigación que respalda cada trabajo para definir los derechos de autor. 


\section{REFERENCIAS}

[1]. Fluid Controls. (2017). Pressure Regulator Function | Types of Pressure Regulators. [online] Disponible en: http://www.fluidcontrols.co.uk/how-pressure-regulators-work/

[2]. Pegacor MAX, adhesivo bajo en polvo. (2016). Materiales \& Pinturas Corona. [online] Corona. Disponible en: http://claraluciagomez.com/wp-content/uploads/2016/06/ficha-tecnica-pegacor-max.pdf

[3]. Ccs.org.co. (2015). La Seguridad y Salud en el Trabajo en cifras. [online] Disponible en: http://ccs.org.co/salaprensa/index.php?option=com_content\&view=article\&id=573:sst\&catid=320\&Itemid $=856$

[4]. Consolidado estadísticas accidentes y enfermedades laborales - 2016. (2016). [online] Bogotá: Fondo de Riesgos laborales de la República de Colombia. Disponible en: http://fondoriesgoslaborales.gov.co/seccion/informacion-estadistica/2016.html

[5]. Fasecolda.com. (2016). Fasecolda: Sector enero 28 2016. [online] Disponible en: http://www.fasecolda.com/index.php/sala-de-prensa/noticias/2016/enero1/sector-enero-28-2016/

[6]. F. Mecafenix, "Servomotor ¿Qué es y cómo funciona?”, ingmecafenix, 2017. [Online]. Disponible en: https://www.ingmecafenix.com/electricidad-industrial/servomotor/

[7]. Bos, F., Wolfs, R., Ahmed, Z. and Salet, T. (2016). Additive manufacturing of concrete in construction: potentials and challenges of 3D concrete printing. Virtual and Physical Prototyping, 11(3), pp.209-225.

[8]. Malaeb, Z., Hachem, H., Tourbah, A., Maalouf, T., El Zarwi, N., \& Hamzeh, F. (2015). 3D Concrete Printing: Machine and Mix Design. International Journal of Civil Engineering and Technology, 6, 14-22.

[9]. EA Avallone, IT Baumeister, A Sadegh (2006) Instrument Engineers' Handbook,(Volume 2) Third Edition .pdfs.semanticscholar.org

[10]. A.C. Solé, Neumática e Hidráulica, España: marcombo, 2007. 